\title{
Metapopulation persistence and species spread in river networks
}

\author{
Lorenzo Mari ${ }^{1,2}$, Renato Casagrandi ${ }^{1}$, Enrico Bertuzzo ${ }^{2}$, \\ Andrea Rinaldo ${ }^{2,3}$, Marino Gatto ${ }^{1}$
}

${ }^{1}$ Dipartimento di Elettronica, Informazione e Bioingegneria, Politecnico di Milano, Piazza Leonardo da Vinci 32, 20133 Milano, Italy

${ }^{2}$ Laboratory of Ecohydrology ECHO/IIE/ENAC, École Polytechnique Fédérale de Lausanne, 1015 Lausanne, Switzerland

${ }^{3}$ Dipartimento di Ingegneria Civile, Edile ed Ambientale, Università di Padova, 35131 Padova, Italy

Article type Letter

Running title Metapopulation persistence in river networks

Corresponding Lorenzo Mari (mail: lorenzo.mari@polimi.it;

author phone: +39.02 .2399 .3562 ; fax: $+39.02 .2399 .3412)$

Other $\quad$ RC: renato.casagrandi@polimi.it; EB: enrico.bertuzzo@epfl.ch;

contacts_ AR: andrea.rinaldo@epfl.ch; MG: marino.gatto@polimi.it

Document abstract: 150 words; text: $\sim 5,000$ words;

statistics figures: 4; tables: 1; references: 49

Keywords ecohydrology, metapopulation capacity, extinction debt, topology, fluvial systems, dominant eigenvalue, bifurcations, movement ecology

Statement of All authors contributed substantially to designing research, LM and MG authorship performed research, EB and AR provided hydrological tools, LM and RC wrote the first draft of the ms, all authors contributed to revising the ms 


\begin{abstract}
River networks define ecological corridors characterized by unidirectional streamflow, which may impose downstream drift to aquatic organisms or affect their movement. Animals and plants manage to persist in riverine ecosystems, though, which in fact harbor high biological diversity. Here we study metapopulation persistence in river networks analyzing stage-structured populations that exploit different dispersal pathways, both along-stream and overland. Using stability analysis we derive a novel criterion for metapopulation persistence in arbitrarily complex landscapes described as spatial networks. We show how dendritic geometry and overland dispersal can promote population persistence, and that their synergism provides an explanation for the so-called 'drift paradox'. We also study the geography of the initial spread of a species and place it in the context of biological invasions. Applications concerning the persistence of stream salamanders in the Shenandoah river, and the spread of two invasive species in the Mississippi-Missouri are also discussed.
\end{abstract}

\title{
1 Introduction
}

Population persistence in riverine streams represents a long-standing issue in freshwater ecology (Speirs \& Gurney, 2001; Lutscher et al., 2010). These ecosystems are in fact characterized by strong unidirectional water flow, which imposes downstream drift to the movement of aquatic organisms. In the absence of mechanisms allowing for upstream colonization, the persistence of riverine populations would hardly be possible. This 'drift paradox' is especially relevant to non-sessile organisms with low self-propelled motion capacity, such as the larval stages typical of many freshwater species. To explain the long-term persistence of such populations several mechanisms have been proposed. As first empirically documented by Müller (1954) in Scandinavian freshwater ecosystems, many insect species compensate larval drift with upstream-directed flight of adults prior to oviposition (Müller's colonization 
cycle; see Müller, 1982). As an alternative explanation, Waters (1972) proposed an excess production hypothesis, in which drifting organisms are supposed to be those who exceed the balance of numbers at the local scale (thus implicitly assuming that drift represents an extra-mortality term). Also, hydrodynamic stream heterogeneities have been shown to cause organism retention in hydrodynamic in-flow refugia (e.g. Reynolds et al., 1991; Lancaster \& Hildrew, 1993a,b).

The passive movement of an aquatic organism in a river system mainly results from the combination of advection, as determined by the dominant uniform streamflow, and diffusion, as determined by local streamflow heterogeneities. Active movement, occurring via swimming, crawling or flying (either directly or through some dispersal vector) further increases macroscopic diffusion - in some cases becoming the predominant source of motion. These factors act within a distinctive landscape topology, usually characterized by a hierarchical branching geometry endowed with universal scaling features (Rodriguez-Iturbe \& Rinaldo, 1997). Riverine ecosystems are in fact among the most representative examples of dendritic ecological networks (Peterson et al., 2013). Diffusive dispersal (Speirs \& Gurney, 2001; Pachepsky et al., 2005) and river network topology (Fagan, 2002; Campbell Grant, 2011) have been recently proposed as key factors for the persistence of riverine populations. Topology is particularly important in constraining the dispersal of aquatic species lacking life stages that can disperse overland (Fagan, 2002). This mode of dispersal has been both theoretically postulated (Lutscher et al., 2005) and experimentally observed (Carrara et al., 2012) to facilitate the persistence of riverine populations. Dispersal can occur at different life stages, most frequently early in the life history of aquatic organisms. As an example, in a massive mark-recapture study (Campbell Grant et al., 2010) of two lungless salamander species in stream networks of Virginia, the newly metamorphosed (juvenile) salamanders had the highest probabilities of dispersing to other stream reaches, thus being the primary responsible for overland connections. While it is relatively common to find freshwater organ- 
isms that begin their life cycle as motile and reach maturity as sessile (e.g. mussels), there are notable exceptions - as in the case of parasites with complex life cycles that involve intermediate hosts with low motility (e.g. snails) and final hosts with high motility (e.g. fish; see Blasco-Costa et al., 2012).

Despite their importance, diffusive dispersal, landscape geometry, stage-dependent movement and exploitation of multiple dispersal pathways have not yet been analyzed together to yield a comprehensive description of the conditions leading to the persistence and spread of riverine populations. Classical approaches in fact include the analysis of reaction-advectiondiffusion (Speirs \& Gurney, 2001; Pachepsky et al., 2005), integro-differential (Lutscher et al., 2005) or integro-difference equations (Lutscher et al., 2010) in simple one-dimensional (1-D) landscapes. Dendritic geometries have been considered in simulation studies of individualbased models (Fagan, 2002; Campbell Grant, 2011) and in matrix population models applied to stage-structured populations in networks of habitat patches (Goldberg et al., 2010). This study is particularly interesting because it is devoted to the analysis of branching spatial structure and life history on the asymptotic growth rate of a riverine population - with clear implications for population persistence. Also, some analytical results for the persistence of a population subject to advection and diffusion on a tree graph have been provided recently by Ramirez (2012).

Here, we use stability analysis to derive a spatially explicit persistence criterion for riverine metapopulations. A technically similar approach has recently been used in an epidemiological context to determine invasion thresholds for pathogens of waterborne diseases (Gatto et al., 2012, 2013). We analyze a model network for a riverine metapopulation with two developmental stages (juveniles and adults) in which individuals are allowed to move according to multiple stage-dependent dispersal pathways, typically along the hydrological network and overland. We use the criterion to study the effects of contrasting geometries and different ecohydrological conditions on metapopulation persistence. We also analyze a 
case study pertaining the persistence of a metapopulation of stream salamanders inhabiting the Shenandoah river (Virginia, US). As the conditions for metapopulation persistence in a river ecosystem correspond to those for species spread, we discuss how to use our framework in the context of biological invasions, for which the analysis of dispersal pathways is crucial to focusing mitigation and conservation efforts. To that end, we study retrospectively two recent biological invasions of a large fluvial system (namely, the spread of the zebra mussel and of the Asian carp in the Mississippi-Missouri river system, MMRS).

\section{Material and methods}

\section{The model}

We consider a prototypical aquatic metapopulation living in a river network made of $n$ nodes (Fig. 1a), each of which represents a river stretch where local ecological conditions are identical. We assume that the species has two ecologically distinct developmental stages, thus we split the population in young (non-reproductive) individuals $(Y)$ and adult (reproductive) individuals $(A)$. Movement from node to node can occur through different pathways, either along the stream network or overland. Local demographic processes (birth, growth and death) and dispersal dynamics in each node $i$ of the river network are described by the following system of $2 n$ ordinary differential equations

$$
\begin{aligned}
\frac{d Y_{i}}{d t} & =-\mathcal{M}_{Y}\left(Y_{i}, A_{i}\right) Y_{i}-\gamma Y_{i}+\mathcal{N}\left(Y_{i}, A_{i}\right) A_{i}-\sum_{h=1}^{N_{Y}} l_{h}\left(Y_{i}-\sum_{j=1}^{n} P_{h j i} Y_{j}\right) \\
\frac{d A_{i}}{d t} & =-\mathcal{M}_{A}\left(Y_{i}, A_{i}\right) A_{i}+\gamma Y_{i}-\sum_{k=1}^{N_{A}} m_{k}\left(A_{i}-\sum_{j=1}^{n} Q_{k j i} A_{j}\right),
\end{aligned}
$$

where: $\mathcal{M}_{Y}\left(Y_{i}, A_{i}\right)\left[\mathcal{M}_{A}\left(Y_{i}, A_{i}\right)\right]$ is the (possibly) density-dependent per-capita mortality rate for juveniles [adults]; $\gamma$ is the rate at which young individuals become adult $(1 / \gamma$ thus being 
the average duration of the juvenile phase); $\mathcal{N}\left(Y_{i}, A_{i}\right)$ is the (possibly) density-dependent natality rate of adults; $l_{h}\left[m_{k}\right]$ is the rate at which young [adult] organisms undergo dispersal along to the $h$-th $[k$-th $]$ pathway $\left(h=1 . . N_{Y}\left[k=1 . . N_{A}\right]\right.$ being the number of possible dispersal mechanisms for juveniles [adults]); and $P_{h_{j i}}\left[Q_{k_{j i}}\right]$ is the fraction of young [adult] organisms moving from node $j$ to node $i$ through the $h$-th $[k$-th] dispersal mechanism available to juveniles [adults]. We assume that the mortality [natality] rate is a monotonically increasing [decreasing] function of population density $\left(\partial \mathcal{M}_{Y, A} / \partial\left(Y_{i}, A_{i}\right) \geq 0, \partial \mathcal{N} / \partial\left(Y_{i}, A_{i}\right) \leq 0\right.$ for any $i$ ), i.e. that there is no depensation or Allee effect. Note that the assumption of spatial homogeneity of the parameters can be relaxed, and that the model can also be easily extended to describe populations with more complex age/stage structures. A comprehensive list of mathematical symbol is reported in Table 1.

\section{Figure 1 and Table 1 about here}

\section{Connectivity structures and dispersal mechanisms}

Dispersal probabilities $P_{h_{j i}}$ and $Q_{k_{j i}}$ depend on the connectivity structure provided by the environmental matrix and the dispersal mechanisms relevant to the metapopulation being investigated. As for connectivity, we consider three hypothetical network structures for theoretical analyses and two real river networks (the Shenandoah river and the MMRS) for more realistic case studies. The hypothetical networks considered here are a 1-D lattice (Fig. 1b), a deterministic fractal, namely a Peano construct (Fig. 1c) and a so-called Optimal Channel Network (OCN, Fig. 1d; Rodriguez-Iturbe \& Rinaldo, 1997). While the lattice geometry clearly represents an oversimplification of real river networks (although widely used to study population persistence in riverine ecosystems; see e.g. Speirs \& Gurney, 2001; Lutscher et al., 2005), Peano's topological measures match closely those of real river networks. OCNs represent a further step forward, in that their topological and metric properties are 
virtually undistinguishable from those of real river networks.

As for dispersal pathways, the first and foremost mechanism to be considered in a riverine setting is along-stream aquatic dispersal, which may describe both hydrological drift and active movement along river corridors (see Appendix S1 in Supporting Information). Other mechanisms can be relevant to the dispersal of riverine populations as well. For instance, flying or human/animal-mediated transport processes (Wilson et al., 2009) could be only partially constrained by river network geometry and flow direction (see e.g. Collier \& Smith, 1998; Didham et al., 2012, for empirical evidence concerning insect flight), thus potentially providing aquatic organisms with suitable pathways for unbiased overland dispersal. This can be described by e.g. an exponential kernel (Hanski \& Ovaskainen, 2000), but other, possibly ad hoc, mechanisms can obviously be introduced to describe dispersal in speciesspecific case studies.

Dispersal probabilities are subsumed into connection matrices, namely $\mathbf{P}_{\mathbf{h}}=\left[P_{h i j}\right]$ and $\mathbf{Q}_{\mathbf{k}}=\left[Q_{k_{i j}}\right]$. We assume that $\sum_{j=1}^{n} P_{h i j} \leq 1$ and $\sum_{j=1}^{n} Q_{k_{i j}} \leq 1$ for any $i, h$ and $k$. Specifically, row-wise sums can be less than one in the presence of absorbing boundary conditions (see again Appendix S1) and/or costly dispersal (Casagrandi \& Gatto, 1999), which both imply the non-conservation of the abundance of dispersing organisms. Finally, the union of the graphs associated with the matrices $\mathbf{P}_{\mathbf{h}}$ and $\mathbf{Q}_{\mathbf{k}}$ is assumed to be strongly connected, so that it is always possible for the individuals of the focal species to find a path between any two nodes of the river network via the available dispersal pathways.

\section{Derivation of persistence conditions}

Irrespectively of parameter values, the state $\mathbf{X}_{\mathbf{0}}$ characterized by $Y_{i}=0$ and $A_{i}=0$ for any $i$ is a global extinction equilibrium for model (1). In the absence of an Allee effect, metapopulation persistence is related to the stability of this equilibrium. In fact, if $\mathbf{X}_{\mathbf{0}}$ is stable the population cannot persist in any of the river network nodes. On the contrary, if 
$\mathbf{X}_{\mathbf{0}}$ is unstable, juvenile and adult abundances, even if initially small, are expected to grow - thus granting metapopulation persistence. The condition for the extinction equilibrium to switch from stable to unstable is that the Jacobian matrix $\mathbf{J}$ of system (1) linearized at $\mathbf{X}_{\mathbf{0}}$ has one zero eigenvalue. Population persistence can thus be assessed by analyzing how the eigenvalues of $\mathbf{J}$ vary with model parameters, connectivity structures and dispersal mechanisms.

\section{Spatial patterns of species spread}

In our framework, the condition under which a species can invade a river network corresponds to that for metapopulation persistence. As such, if the global extinction equilibrium is unstable, the dominant eigenvector of matrix $\mathbf{J}$ pinpoints the direction in the state space along which the system trajectories, after a transient period due to initial conditions, will diverge from the equilibrium. Specifically, the components of the leading eigenvector correspond to the evolving abundances of young or adult individuals in different locations of the river network. The analysis of the dominant eigenvector of the Jacobian of system (1) evaluated at $\mathbf{X}_{\mathbf{0}}$ is thus key to understanding the early spatial patterns of species spread, and can thus be useful - at least from a qualitative perspective - to study the geography of aquatic invasions in riverine ecosystems.

\section{Results}

\section{A spatially explicit persistence criterion}

As detaild in Appendix S2, the stability switch of the extinction equilibrium corresponds to the condition $\operatorname{det}\left(\mathbf{I}_{\mathbf{n}}-\mathbf{J}^{\star}\right)=0$, where $\mathbf{J}^{\star}$ is a matrix of size $n$, deducible from the $2 n$-sized 
Jacobian $\mathbf{J}$ of (1), defined as

$$
\begin{aligned}
\mathbf{J}^{\star} & =R_{0} \mathbf{I}_{\mathbf{n}}+\frac{1}{\mu_{Y}+\gamma} \sum_{h=1}^{N_{Y}} l_{h}\left(\mathbf{P}_{\mathbf{h}}^{T}-\mathbf{I}_{\mathbf{n}}\right)+\frac{1}{\mu_{A}} \sum_{k=1}^{N_{A}} m_{k}\left(\mathbf{Q}_{\mathbf{k}}^{T}-\mathbf{I}_{\mathbf{n}}\right)+ \\
& -\frac{1}{\mu_{A}\left(\mu_{Y}+\gamma\right)} \sum_{h=1}^{N_{Y}} l_{h}\left(\mathbf{P}_{\mathbf{h}}^{T}-\mathbf{I}_{\mathbf{n}}\right) \sum_{k=1}^{N_{A}} m_{k}\left(\mathbf{Q}_{\mathbf{k}}^{T}-\mathbf{I}_{\mathbf{n}}\right) .
\end{aligned}
$$

In the previous expression, $R_{0}=\nu \gamma / \mu_{A} /\left(\mu_{Y}+\gamma\right)$ is the quantity controlling population persistence in a non-spatial setting and can be interpreted as the average number of daughters successfully reaching maturity generated by one mother during her entire lifetime. The condition for an isolated population to persist is thus $R_{0}>1$. In the presence of dispersal, instead, metapopulation persistence is determined by the dominant eigenvalue $\lambda_{\max }\left(\mathbf{J}^{\star}\right)$. Specifically, the persistence-extinction boundary (i.e. the curve or surface in the system parameter space that separates parameter combinations corresponding to metapopulation extinction from those corresponding to persistence; Casagrandi \& Gatto, 1999) is given by the condition

$$
E_{0}=\lambda_{\max }\left(\mathbf{J}^{\star}\right)>1
$$

In other words, the occasional introduction of some individuals in some network nodes results in a successful colonization if (and only if) $E_{0}>1$. In this case, the assumption of strong connectivity made above implies that persistence is granted in all the network nodes.

Criterion (3), shows that not only local demographic processes (first term in the righthand side of eq. (2)), but also average net immigration from connected sites (second and third terms) is relevant to the persistence of riverine metapopulations. It also shows that the intertwining between different dispersal pathways may have nontrivial effects on metapopulation persistence or extinction (last term in the right-hand side of eq. (2)). As a matter of fact, the persistence condition is based on the dominant eigenvalue of $\mathbf{J}^{\star}$, which is not simply deducible from $R_{0}$ and the eigenvalues of matrices $\mathbf{P}_{\mathbf{h}}$ and $\mathbf{Q}_{\mathbf{k}}$. Note that, close to the 
persistence-extinction boundary, $E_{0}$ also sets a timescale for metapopulation dynamics and, in particular, for metapopulation extinction (see again Appendix S2). Criterion (3) can be extended to account for spatial heterogeneities in the model parameters whenever relevant for the underlying ecological processes (Appendix S3).

\section{The role of network structure and dispersal pathways}

As a basic test case to study persistence in a river network, we have analyzed a population in which adults are sessile and juveniles are subject to drift/operate along-stream dispersal $\left(l_{1}>\right.$ $0, l_{h}=0$ for any $h>1, m_{k}=0$ for any $k, \mathbf{P}_{\mathbf{1}}=\mathbf{F}$, with $\mathbf{F}$ being the hydrological connection matrix; see Appendix S1). Fig. 2a (gray lines) shows that high values of along-stream dispersal and bias are always detrimental to species persistence, and that network topology remarkably influences the fate of the metapopulation. Specifically, more complex networks (Peano, OCN) favor metapopulation persistence compared to simpler geometries (lattice) with the same backbone length. Quite interestingly, the largest relative differences emerging from the three contrasting topologies are found for high dispersal rates and low values of the transport bias. In these conditions, along-stream movement is significantly influenced by geomorphological dispersion, i.e. by the intertwining of hydrodynamic dispersion within individual reaches and the morphology of the network structure (Rinaldo et al., 1991).

\section{Figure 2 about here}

Changes in the flow regime can obviously affect the persistence of metapopulation dispersing through water pathways. Fig. 2b (gray lines) shows that the metapopulations that cannot compensate higher bias of aquatic dispersal with higher natality are doomed to extinction - i.e. that downstream drift reduces metapopulation capacity (sensu Hanski \& Ovaskainen, 2002). Close to the persistence-extinction boundary the dynamics of the metapopulation is very slow, because $E_{0} \approx 1$ (and $\lambda_{\max }(\mathbf{J}) \approx 0$ ). Therefore, extinctions may occur over 
long (yet still ecological) timescales depending on the distance from the bifurcation curve characterized by $E_{0}=1$. This delay generates an extinction debt (sensu Tilman et al., 1994). As an example, the model predicts extinction for all the parameter settings lying below the persistence-extinction boundaries in Fig. $2 \mathrm{~b}$ - yet in the light-gray-shaded regions metapopulation extinction will take more than 10 years, approximately corresponding to 10 generation times for the population under study (see again Appendix S2).

To analyze how different dispersal pathways can influence metapopulation persistence, we have studied populations in which juveniles disperse not only along the hydrological network but also overland $\left(l_{1}>0, l_{2}>0, l_{h}=0\right.$ for any $h>2, m_{k}=0$ for any $k$, $\mathbf{P}_{\mathbf{1}}=\mathbf{F}, \mathbf{P}_{\mathbf{2}}=\mathbf{G}$, with $\mathbf{G}$ being the connection matrix describing overland isotropic dispersal with characteristic dispersal length $D$; see Appendix S1). Figs. 2a and 2b (black lines) show that overland dispersal can remarkably benefit riverine metapopulation persistence, in particular for high values of the bias of along-stream dispersal. Under these conditions, corresponding in fact to advection-dominated environments, overland dispersal can provide riverine populations with an effective mean of upstream propagation, thus mitigating the downstream drift imposed to offspring and juveniles by passive hydrological transport. These results hold qualitatively for all the considered network topologies (not necessarily riverine; see Appendix S4 for some examples of 2-D lattice geometries). However, it is apparent that topological complexity and the multiplicity of dispersal pathways operate synergistically (last term in eq. (2)), thus greatly favoring the persistence of metapopulation inhabiting complex river networks (Fig. 2c). The effects of this synergism are very robust not only to changes of the demographic rates, but also to variations in the exploitation of different dispersal pathways in relation to specific life histories (Appendix S5).

One might wonder whether enhanced persistence due to the superimposition of different dispersal pathways is simply due to higher overall (i.e. along-stream + overland) dispersal. We have thus repeated some of the analyses above considering different dispersal strategies, 
defined as the combination of overland and aquatic dispersal operated by a population.

Specifically, we assume that a fraction $\phi$ of the total movement rate $K$ is allocated to overland movement, while the remaining fraction $1-\phi$ is allocated to water-mediated dispersal. Fig. $2 \mathrm{~d}$ reports a systematic exploration of the parameter space $(K, \phi)$, each point of which represents a different dispersal strategy, and shows that even relatively small fractions of total movement rate allocated to overland dispersal are sufficient to guarantee persistence. The exploitation of alternative dispersal pathways (specifically, of overland dispersal) can thus remarkably affect the fate of a population subject to downstream drift in a riverine ecosystem.

\section{Persistence of an amphibian metapopulation in a river system}

The framework presented above can be adapted to study the persistence of a real metapopulation in a river network. As a proof of concept, here we study the fate of a metapopulation of stream salamanders in the Shenandoah river network (Virginia, US; Fig. 3a). Model (1) has been parameterized with demographic (Organ, 1961) and dispersal (Campbell Grant et al., 2010) data relative to the salamander species Desmognathus fuscus and D. monticola (technical details in Appendix S6). The juveniles of these two amphibian species can move both along stream corridors and overland, while larvae and adults are almost sessile. Quite interestingly, juveniles' along-stream dispersal is known to be biased towards upstream sites in the river network. Despite the ongoing decline of amphibian abundances worldwide, populations of stream salamanders in Eastern North America are reportedly stable - an observation that has been linked to their ability to exploit multiple dispersal pathways (see again Campbell Grant et al., 2010).

Our analysis shows that if the cost of dispersal (here defined as the fraction of individuals that disperse outside their suitable habitat) is negligible, both along-stream (low $\epsilon_{s}$ ) and overland (low $\left.\epsilon_{o}\right)$, then the salamander metapopulation is predicted to persist $\left(E_{0} \gg 1\right)$. 
However, for increasing values of the cost of dispersal (possibly due to the alteration of the habitat template), the metapopulation can cross the persistence-extinction boundary, and can thus be doomed to extinction (Fig. 3b). It is also possible to test the sensitivity of $E_{0}$ to changes of the model parameters. Besides expected positive [negative] effects of increased natality $\nu$ [mortality $\mu_{Y}$ and $\mu_{A}$ ] on $E_{0}$, increasing levels of overland dispersal $l_{2}$ can promote metapopulation persistence (as suggested by Campbell Grant et al., 2010), provided that the cost of overland movement is lower than that of along-stream dispersal. $E_{0}$ can actually peak for intermediate values of the overland dispersal rate (as in Fig. 3c), a result that mirrors the intermediate dispersal principle of metapopulation ecology (e.g. Casagrandi \& Gatto, 1999, 2006).

\section{The geography of riverine biological invasions}

The dominant eigenvector of the linearized model nearby extinction is a synthetic spatial indicator of the initial spread of a riverine population, not only in theoretical settings (as in Appendix S7) but also in real applications, as it turns out by adapting model (1) to qualitatively describe two paradigmatic examples of biological invasion recorded in the MMRS (Fig. 4a). Here we report the results for the first example only (the zebra mussel colonization of the MMRS; technical details in Appendix S8), while the second example (the Asian carp invasion of the MMRS) is discussed in Appendix S9.

The zebra mussel (Dreissena polymorpha), a freshwater bivalve native to Eurasia, invaded the Great Lakes region in the late 1980's and rapidly spread across North America inland waters (e.g. Strayer, 2009, see also panels b and c of Fig. 4). The main vectors of species dispersal during the early phase of the invasion were hydrological transport of larvae (the so-called 'veligers') and long-distance port-to-port veliger dispersal because of inland com- 
mercial navigation (Mari et al., 2011). The veligers may in fact be shipped within the ballast water of commercial vessels, thus allowing the species to disperse over very long distances and to colonize stretches of the river network that could not have been reached otherwise.

\section{Figure 4 about here}

We have parameterized model (1) to describe zebra mussel invasion dynamics (Casagrandi et al., 2007; Mari et al., 2009, 2011), namely by considering sessile adults and a juvenile stage during which veligers can undergo dispersal through hydrological transport and port-to-port relocation (described by a port-to-port connectivity matrix $\Gamma$ with characteristic dispersal distance $\Delta$; see Appendix S8). The resulting value of $E_{0} \gg 1$ implies that the zebra mussel can persist and spread in the MMRS (as indeed observed; note that a high value of $E_{0}$ corresponds to a great risk of invasion). The dominant eigenvector of the linearized model computed under these hypotheses turns out to be a satisfactory qualitative indicator of the risk of mussel spread in the early phase of the invasion (Fig. 4d). Interestingly, the dominant eigenvector of matrix $\mathbf{J}$ correctly predicts the widespread occurrence of zebra mussel colonies throughout the MMRS, not only in the Upper and Lower Mississippi, but also in the lower course of the Missouri river, as well as in the Ohio river - i.e. in the rivers where most of the largest MMRS commercial ports are located (see also Fig. 2a in Mari et al., 2011). Numerical experiments suggest that the dominant eigenvector of matrix $\mathbf{J}$ is also rather a robust descriptor of the spatial patterns of early population spread, even in the presence of non-negligible uncertainty in the model parameters (see again Appendix S7).

\section{Discussion}

In this work we have proposed a novel way to determine conditions for population persistence and spread in a river network. Our study has shown that metapopulation persistence is 
determined by complex interactions between network geometry and dispersal mechanisms among which along-stream movement plays a prominent role in fluvial settings. In particular, we have shown that a dendritic geometry does enhance metapopulation persistence in a river network. This result echoes recent theoretical and experimental findings that have linked dendritic topologies to long species persistence times and high local biodiversity (e.g. when compared to 2-D landscapes; Bertuzzo et al., 2011; Carrara et al., 2012; Suweis et al., 2012; Altermatt, 2013). Our work has also formally confirmed that overland dispersal can favor metapopulation persistence, especially (but not only) for species subject to hydrological drift. Moreover, extra-range dispersal, i.e. the movement of organisms from the current range to a new area of suitable habitat (e.g. Wilson et al., 2009), is expected to influence both metapopulation persistence and spatiotemporal invasion patterns, as indeed found in the zebra mussel invasion of the MMRS. We can thus conclude that diffusive dispersal, landscape geometry, and exploitation of multiple dispersal pathways may offer a multifaceted solution of the 'drift paradox' for riverine populations. More in general, our work shows that these ingredients are key to understanding metapopulation persistence in realistic (or even real) landscapes.

Although derived in the context of river systems, the persistence criterion proposed in this work can be adapted to populations living in different ecosystems, possibly characterized by high levels of spatial complexity. As an example, an interesting application would be the analysis of 2-D lattice geometries, which would allow to address the study of persistence conditions for terrestrial metapopulations. Preliminary explorations (Appendix S4) confirm that, also in 2-D lattices, metapopulation persistence is deeply related to the connectivity of the underlying environmental matrix, as well as to the dispersal mechanisms relevant to the metapopulation. The flexibility of our tools is essentially granted by the multi-layer network framework (Mari et al., 2011), which generalizes previous network-based approaches in metapopulation ecology (e.g. Hanski \& Ovaskainen, 2000) and allows a hier- 
archical description of the interactions between ecological and spatial dynamics at different level of organizational complexity. In addition, our mathematical framework can be readily applied to real case studies whenever there is sufficient information on the focal species to: i) formalize a demographic model for its local-scale dynamics; ii) identify the underlying environmental matrix constituting its habitat (such as river stretches in a fluvial system, patches in a fragmented forest, etc.); iii) sort out its main dispersal pathways (e.g. Appendices 6, 8 and 9). In the absence of detailed information on the dynamics of the focal population (which is often the case for endangered species), scaling relations could assist in the definition of its demographic parameters (Marquet et al., 2005). The model can then guide the analysis of persistence conditions for metapopulations living in realistic ecosystems, possibly subject to habitat alterations.

Human activities represent a main driver for such alterations. Damming, for instance, is usually cited as a primary threat to the integrity of riverine habitats (see e.g. Allan \& Castillo, 2007). From an ecological perspective, one of the main effects of damming (in addition to changes of water quality and assemblage composition) is that of reducing alongstream dispersal and migration, especially in the upstream direction. This would in turn entail a highly biased hydrological dispersal. Our analysis has shown that increasing bias could reduce metapopulation capacity (Hanski \& Ovaskainen, 2002), i.e. it could doom to extinction species that rely on aquatic dispersal and that cannot compensate higher drift with higher natality. In contrast, species that can disperse overland at some specific life stage are predicted to be more resilient to environmental changes, such as alterations of the flow regime or habitat fragmentation. Extinction debts and average times to metapopulation extinction (also known as relaxation times; see e.g. Tilman et al., 1994; Hanski \& Ovaskainen, 2002; Kuussaari et al., 2009; Hylander \& Ehrlén, 2013) can also be quantified through the analysis of persistence-extinction boundaries.

The presented framework could obviously be made even more realistic in many respects. 
In its present form, for instance, it does not account for the possible temporal variability of the environmental conditions which, however, has already been proposed - along with spatial heterogeneity - as an important factor for population persistence in advective environments (e.g. Speirs \& Gurney, 2001; Lutscher et al., 2006; Lutscher \& Seo, 2011). Incorporating spatial heterogeneity in the model parameters is relatively straightforward and does bear major changes to our derivation of persistence conditions - although the algebra required is rather involved (Appendix S3). On the contrary, adding seasonal variability would demand a considerably more elaborated mathematical treatment, possibly relying on Floquet (e.g. Klausmeier, 2008) or Lyapunov exponents (e.g. Ferrière \& Gatto, 1995). Another aspect that certainly deserves future investigations is demographic stochasticity, that has already been shown to play an important role for metapopulations dynamics close to the extinction threshold (e.g. Casagrandi \& Gatto, 1999, 2006).

Despite its limitations, the theoretical framework used to derive persistence conditions (i.e. the stability analysis of an ordinary differential equation network model) can be applied to study other ecological problems. We envisage that similar persistence criteria could in fact be usefully applied to design natural reserves aimed at preserving ecologically important species, as already proposed for marine protected areas (White et al., 2010; Aiken \& Navarrete, 2011) and fragmented landscapes (Hanski \& Ovaskainen, 2000). In these cases, too, metapopulation persistence can be established by properly accounting for the relevant spatial interactions and studying the conditions under which the extinction equilibrium changes its stability properties. Eigenvector analysis could then assist in designing spatially-calibrated conservation efforts. In an even broader perspective, extending our framework to interacting functional groups would allow to study persistence of aquatic metacommunities (rather than metapopulations). With functional diversity being tightly related to ecosystem functioning and services (Naeem et al., 2012), achieving a better understanding of how we can preserve it through suitably targeted actions would certainly represent a major accomplishment for 
current conservation ecology.

\section{Acknowledgments}

LM, EB and AR acknowledge the support provided by ERC advanced grant program through the project RINEC-227612, and by the SFN/FNS project 200021_124930 and 200020_140661.

\section{References}

1.

Aiken, C. \& Navarrete, S. (2011). Environmental fluctuations and asymmetrical dispersal: generalized stability theory for studying metapopulation persistence and marine protected areas. Marine Ecology Progress Series, 428, 77-88.

2 .

Allan, J. \& Castillo, M. (2007). Stream Ecology. Springer, Dordrecht, The Netherlands.

3.

Altermatt, F. (2013). Diversity in riverine metacommunities: a network perspective. Aquatic Ecology, 47, 365-377.

4.

Bertuzzo, E., Suweis, S., Mari, L., Maritan, A., Rodriguez-Iturbe, I. \& Rinaldo, A. (2011). Spatial effects on species persistence and implications for biodiversity. Proceedings of the National Academy of Sciences USA, 108, 4346-4351.

5.

Blasco-Costa, I., Waters, J.M. \& Poulin, R. (2012). Swimming against the current: genetic structure, host mobility and the drift paradox in trematode parasites. Molecular Ecology, 21, 207-271. 
6.

Campbell Grant, E. (2011). Structural complexity, movement bias, and metapopulation extinction risk in dendritic ecological networks. Journal of the North American Benthological Society, 30, 252-258.

7.

Campbell Grant, E., Nichols, J., Lowe, W. \& Fagan, W. (2010). Use of multiple dispersal pathways facilitates amphibian persistence in stream networks. Proceeding of the National Academy of Sciences USA, 107, 6936-6940.

8.

Carrara, F., Altermatt, F., Rodriguez-Iturbe, I. \& Rinaldo, A. (2012). Dendritic connectivity controls biodiversity patterns in experimental metacommunities. Proceedings of the National Academy of Sciences USA, 109, 5761-5766.

9.

Casagrandi, R. \& Gatto, M. (1999). A mesoscale approach to extinction risk in fragmented habitats. Nature, 400, 560-562.

10.

Casagrandi, R. \& Gatto, M. (2006). The intermediate dispersal principle in spatially explicit metapopulations. Journal of Theoretical Biology, 239, 22-32.

11.

Casagrandi, R., Mari, L. \& Gatto, M. (2007). Modelling the local dynamics of the zebra mussel (Dreissena polymorpha). Freshwater Biology, 52, 1223-1238.

12.

Collier, K. \& Smith, B. (1998). Dispersal of adult caddisflies (Trichoptera) into forests alongside three New Zealand streams. Hydrobiologia, 361, 53-65. 
13.

Didham, R., Blakely, T., Ewers, R., Hitchings, T., Ward, J. \& Winterbourn, M. (2012). Horizontal and vertical structuring in the dispersal of adult aquatic insects in a fragmented landscape. Fundamental and Applied Limnology, 180, 27-40.

14.

Fagan, W. (2002). Connectivity, fragmentation, and extinction risk in dendritic metapopulations. Ecology, 83, 3243-3249.

15.

Ferrière, R. \& Gatto, M. (1995). Lyapunov exponents and the mathematics of invasion in oscillatory or chaotic populations. Theoretical Population Biology, 48, 126-171.

16.

Gatto, M., Mari, L., Bertuzzo, E., Casagrandi, R., Righetto, L., Rodriguez-Iturbe, I. \& Rinaldo, A. (2012). Generalized reproduction numbers and the prediction of patterns in waterborne disease. Proceedings of the National Academy of Sciences USA, 109, 1970319708.

17.

Gatto, M., Mari, L., Bertuzzo, E., Casagrandi, R., Righetto, L., Rodriguez-Iturbe, I. \& Rinaldo, A. (2013). Spatially explicit conditions for waterborne pathogen invasion. The American Naturalist, 182, 328-346.

18.

Goldberg, E., Lynch, H., Neubert, M. \& Fagan, W. (2010). Effects of branching spatial structure and life history on the asymptotic growth rate of a population. Theoretical Ecology, 3, 137-152. 
19.

Hanski, I. \& Ovaskainen, O. (2000). The metapopulation capacity of a fragmented landscape. Nature, 404, 755-758.

20.

Hanski, I. \& Ovaskainen, O. (2002). Extinction debt at extinction threshold. Conservation Biology, 16, 666-673.

21.

Hylander, K. \& Ehrlén, J. (2013). The mechanisms causing extinction debts. Trends in Ecology and Evolution, 28, 341-346.

22.

Klausmeier, C. (2008). Floquet theory: a useful tool for understanding nonequilibrium dynamics. Theoretical Ecology, 1, 153-161.

23.

Kuussaari, M., Bommarco, R., Heikkinen, R., Helm, A., Krauss, J., Lindborg, R., Öckinger, E., Pärtel, M., Pino, J., Rodà, F., Stefanescu, C., Teder, T., Zobel, M. \& I. Steffan-Dewenter (2009). Extinction debt: a challenge for biodiversity conservation. Trends in Ecology and Evolution, 24, 564-571.

24.

Lancaster, J. \& Hildrew, A. (1993a). Characterising instream flow refugia. Canadian Journal of Fisheries and Aquatic Science, 50, 1663-1675.

25.

Lancaster, J. \& Hildrew, A. (1993b). Flow refugia and the microdistribution of lotic macroinvertebrates. Journal of the North American Benthological Society, 12, 385-393. 
26.

Lutscher, F., Lewis, M. \& McCauley, E. (2006). Effects of heterogeneity on spread and persistence in rivers. Bulletin of Mathematical Biology, 68, 2129-2160.

27.

Lutscher, F., Nisbet, R. \& Pachepsky, E. (2010). Population persistence in the face of advection. Theoretical Ecology, 3, 271-284.

28.

Lutscher, F., Pachepsky, E. \& Lewis, M. (2005). The effect of dispersal patterns on stream populations. SIAM Journal on Applied Mathematics, 65, 1305-1327.

29.

Lutscher, F. \& Seo, G. (2011). The effect of temporal variability on persistence conditions in rivers. Journal of Theoretical Biology, 283, 53-59.

30.

Mari, L., Bertuzzo, E., Casagrandi, R., Gatto, M., Levin, S., Rodriguez-Iturbe, I. \& Rinaldo, A. (2011). Hydrologic controls and anthropogenic drivers of the zebra mussel invasion of the Mississippi-Missouri river system. Water Resources Research, 47, W03523.

31.

Mari, L., Casagrandi, R., Pisani, M., Pucci, E. \& Gatto, M. (2009). When will the zebra mussel reach Florence? a model for the spread of Dreissena polymorpha in the Arno water system (Italy). Ecohydrology, 2, 428-439.

32.

Marquet, P., nones, R.Q., Abades, S., Labra, F., Tognelli, M., Arim, M. \& Rivadeneira, M. (2005). Scaling and power-laws in ecological systems. The Journal of Experimental Biology, 208, 1749-1769. 
33.

Müller, K. (1954). Investigations on the organic drift in North Swedish streams. Tech. rep., Institute of Freshwater Research, Drottningholm.

34.

Müller, K. (1982). The colonization cycle of freshwater insects. Oecologia, 53, 202-207.

35.

Naeem, S., Duffy, J. \& Zavaleta, E. (2012). The functions of biological diversity in an age of extinction. Science, 336, 1401-1406.

36.

Organ, J. (1961). Studies of the local distribution, life history, and population dynamics of the salamander genus Desmognathus in Virginia. Ecological Monographs, 31, 189-220.

37.

Pachepsky, E., Lutscher, F., Nisbet, R. \& Lewis, M. (2005). Persistence, spread and the drift paradox. Theoretical Population Biology, 67, 61-73.

38.

Peterson, E., J.M. Ver Hoef, Isaak, D., Falke, J., Fortin, M., Jordan, C., K. McNyset, Monestiez, P., Ruesch, A., Sengupta, A., Som, N., Steel, E., Theobald, D., Torgersen, C. \& Wenger, S. (2013). Modelling dendritic ecological networks in space: an integrated network perspective. Ecology Letters, 16, 707-719.

39.

Ramirez, J. (2012). Population persistence under advection-diffusion in river networks. Mathematical Biology, 65, 919-942.

40. 
Reynolds, C., Carling, P. \& Beven, K. (1991). Flow in river channels: new insights into hydraulic retention. Archiv für Hydrobiologie, 121, 171-179.

41.

Rinaldo, A., Marani, A. \& Rigon, R. (1991). Geomorphological dispersion. Water Resources Research, 27, 513-525.

42.

Rodriguez-Iturbe, I. \& Rinaldo, A. (1997). Fractal River Basins: Chance and SelfOrganization. Cambridge University Press, New York, NY.

43.

Speirs, D. \& Gurney, W. (2001). Population persistence in rivers and estuaries. Ecology, 82, 1219-1237.

44.

Strayer, D. (2009). Twenty years of zebra mussels: lessons from the mollusk that made headlines. Frontiers in Ecology and Environment, 7, 135-141.

45.

Suweis, S., Bertuzzo, E., Mari, L., Rodriguez-Iturbe, I., Maritan, A. \& Rinaldo, A. (2012).

On species persistence-time distributions. Journal of Theoretical Biology, 303, 15-24.

46.

Tilman, D., May, R., Lehman, C. \& Nowak, M. (1994). Habitat destruction and the extinction debt. Nature, 371, 65-66.

47.

Waters, T. (1972). The drift of stream insects. Annual Review of Entomology, 17, 253-272. 
48.

White, J., Botsford, L., Hastings, A. \& Largier, J. (2010). Population persistence in marine reserve networks: incorporating spatial heterogeneities in larval dispersal. Marine Ecology Progress Series, 398, 49-67.

49.

Wilson, J.R.U., Dormontt, E.E., Prentis, P.J., Lowe, A.J. \& Richardson, D.M. (2009). Something in the way you move: dispersal pathways affect invasion success. Trends in Ecology and Evolution, 24, 136-144. 
Table 1: Mathematical symbols used in the text and their definitions.

\begin{tabular}{|c|c|}
\hline Symbol & Definition \\
\hline & $\underline{\text { State variables }}$ \\
\hline$Y_{i}$ & Abundance of juveniles in node $i$ \\
\hline$A_{i}$ & Abundance of adults in node $i$ \\
\hline & Network geometry \\
\hline$n$ & Number of nodes in the network \\
\hline$n_{b}$ & Number of nodes in the network backbone \\
\hline$L_{b}$ & Length of the network backbone \\
\hline & Demographic parameters \\
\hline $\mathcal{M}_{Y}$ & Density-dependent mortality rate of juveniles \\
\hline$\mu_{Y}$ & Mortality rate of adults at low population density \\
\hline $\mathcal{M}_{A}$ & Density-dependent mortality rate of adults \\
\hline$\mu_{A}$ & Mortality rate of adults at low population density \\
\hline $\mathcal{N}$ & Density-dependent natality rate \\
\hline$\nu$ & Natality rate at low population density \\
\hline$\gamma$ & Rate at which juveniles reach maturity \\
\hline & Dispersal parameters \\
\hline$N_{Y}$ & Number of dispersal pathways available to juveniles \\
\hline$l_{h}$ & Dispersal rate of juveniles along the $h$-th pathway \\
\hline $\mathbf{P}_{\mathbf{h}}$ & Connectivity matrix for the $h$-th juveniles' dispersal pathway \\
\hline$N_{A}$ & Number of dispersal pathways available to adult individuals \\
\hline$m_{k}$ & Dispersal rate of adults along the $k$-th pathway \\
\hline $\mathrm{Q}_{\mathrm{k}}$ & Connectivity matrix for the $k$-th adults' dispersal pathway \\
\hline & Persistence criterion \\
\hline $\mathbf{X}_{0}$ & Extinction equilibrium \\
\hline $\mathbf{I}_{\mathbf{n}}$ & Identity matrix of size $n$ \\
\hline$R_{0}$ & Reproduction number \\
\hline $\mathbf{J}$ & Jacobian matrix of size $2 n$ associated with model (1) (Appendix S2) \\
\hline $\mathbf{J}^{\star}$ & Matrix of size $n$ deducible from $\mathbf{J}$ (Appendix S2) \\
\hline$E_{0}$ & Dominant eigenvalue of matrix $\mathbf{J}^{\star}$ (Appendix S2) \\
\hline & Figure 2 \\
\hline$l_{1}$ & $\overline{\text { Juveniles' along-stream dispersal rate }}$ \\
\hline $\mathbf{F}$ & Hydrological connectivity matrix (Appendix S1) \\
\hline$b$ & Bias of along-stream dispersal (Appendix S1) \\
\hline$l_{2}$ & Juveniles' overland dispersal rate \\
\hline G & Connectivity matrix for overland dispersal (Appendix S1) \\
\hline$D$ & Average distance of the overland dispersal kernel (Appendix S1) \\
\hline$K$ & Total movement rate \\
\hline$\phi$ & Fraction of the total movement rate allocated to overland dispersal \\
\hline & Case studies \\
\hline $\mathbf{N}$ & Connectivity matrix for salamanders' overland dispersal (Appendix S6) \\
\hline$\epsilon_{s}$ & Cost of salamanders' along-stream dispersal (Appendix S6) \\
\hline$\epsilon_{o}$ & Cost of salamanders' overland dispersal (Appendix S6) \\
\hline$\Gamma$ & Port-to-port veligers' connectivity matrix (Appendix S8) \\
\hline$\Delta$ & Average distance of veligers' port-to-port dispersal (Appendix S8) \\
\hline
\end{tabular}




\section{Figure legends}

Figure 1. Schematic representation of the model metapopulation and theoretical river networks used in numerical analyses. (a) A hypothetical sketch of our multi-layer network model (1); hydrological connections (in the example involving juveniles only) are in light gray, while overland connections (in the example adults only) are represented as dashed arrows. (b-d) Different river network topologies; the backbone of each hydrological network has the same number of nodes $\left(n_{b}=33\right)$ and the same arbitrary length $\left(L_{b}=33\right)$ independently of topology, and is indicated by the white-filled nodes (the southmost node being the network outlet).

Figure 2. Persistence conditions for populations with sessile adults and juveniles dispersing via water and overland. Metapopulation persists for parameter combinations below (a) [above $(\mathrm{b}-\mathrm{d})]$ the persistence-extinction boundaries $\left(E_{0}=1\right.$ contour lines, gray and black curves). All rates in year ${ }^{-1}$. (a) Effect of aquatic dispersal parameters without (gray, $l_{2}=0$ ) [with (black, $l_{2}=5$ )] overland dispersal. (b) Effect of transport and demographic parameters in an OCN without (gray) [with (black)] overland dispersal; gray-shaded areas indicate extinction debts longer than 10 years. (c) Effect of overland dispersal parameters $\left(l_{1}=400\right.$, $b=0.9)$. (d) Effect of dispersal strategies $\left(l_{1}=(1-\phi) K, b=0.8, l_{2}=\phi K\right.$, with $\left.K=l_{1}+l_{2}\right)$. Other parameters: $\nu=25, \gamma=1, \mu_{A}=1, \mu_{Y}=5, \mathbf{P}_{\mathbf{1}}=\mathbf{F}, \mathbf{P}_{\mathbf{2}}=\mathbf{G}, D=0.1, l_{h}=0$ for any $h>2, m_{k}=0$ for any $k$.

Figure 3. Persistence of stream salamanders in the Shenandoah river network (Virginia, US). (a) River network geometry; inset: Desmognathus monticola (from USGS). (b) Effect of dispersal cost on population persistence. (c) Sensitivity of $E_{0}$ to variations of the model parameters. Parameters: $\nu=3.9$ juveniles adult $^{-1}$ year $^{-1}, \gamma=0.25, \mu_{Y}=0.40, \mu_{A}=0.92$, $l_{1}=1.58, \mathbf{P}_{\mathbf{1}}=\mathbf{F}, b=-0.49, l_{2}=1.04, \mathbf{P}_{\mathbf{2}}=\mathbf{N}, l_{h}=0$ for $h>2, m_{k}=0$ for any $k$. All rates in year ${ }^{-1}$. See Appendix S6 for details on the parameterization of the model. 
Figure 4. Zebra mussel invasion of the MMRS. (a) River network geometry and localization in the conterminous USA. (b) Fraction of hydrological units (DTAs) invaded by D. polymorpha (inset, from USGS). (c) Spatial occurrence of zebra mussels during the initial phase of the invasion (red in b). (d) Normalized components (adult individuals) of the dominant eigenvector of $\mathbf{J}$. Parameters: $\nu=10^{6}$ larvae adult $^{-1}$ year $^{-1}, \gamma=26, \mu_{Y}=120, \mu_{A}=0.33$, $l_{1}=180, \mathbf{P}_{\mathbf{1}}=\mathbf{F}, b=0.8, l_{2}=3.6, \mathbf{P}_{\mathbf{2}}=\boldsymbol{\Gamma}, \Delta=0.2, l_{h}=0$ for $h>2, m_{k}=0$ for any $k$. All rates in year ${ }^{-1}$. Parameters have been chosen to reproduce the basic timescales of zebra mussels' lifecycle (Appendix S8). 

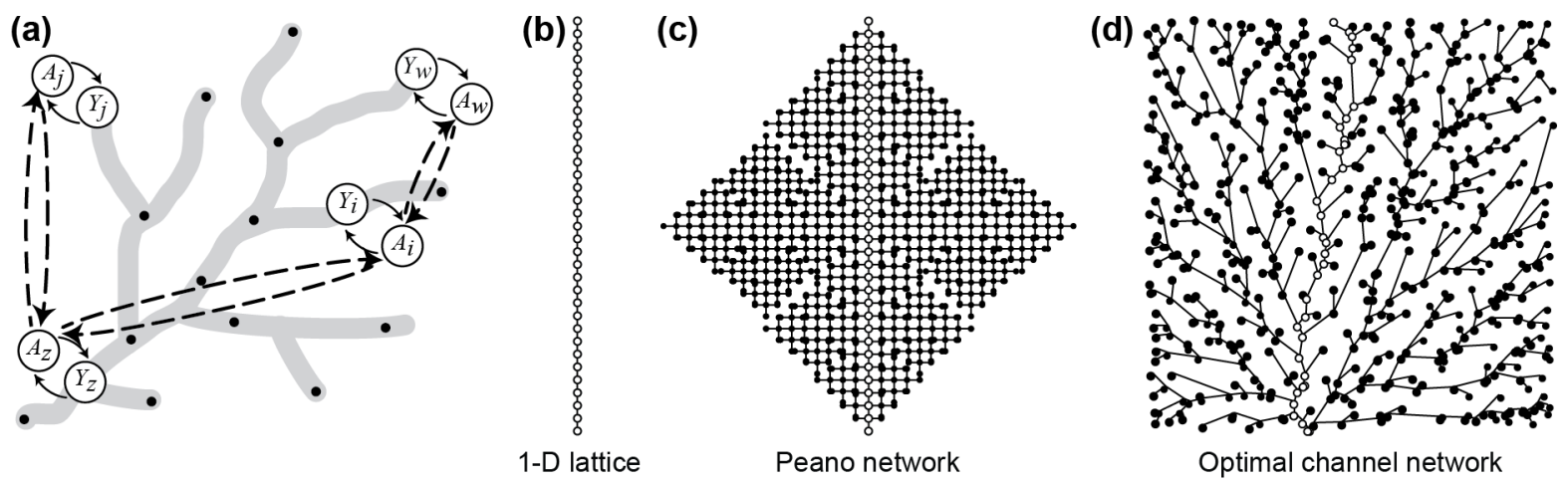

Figure 1:
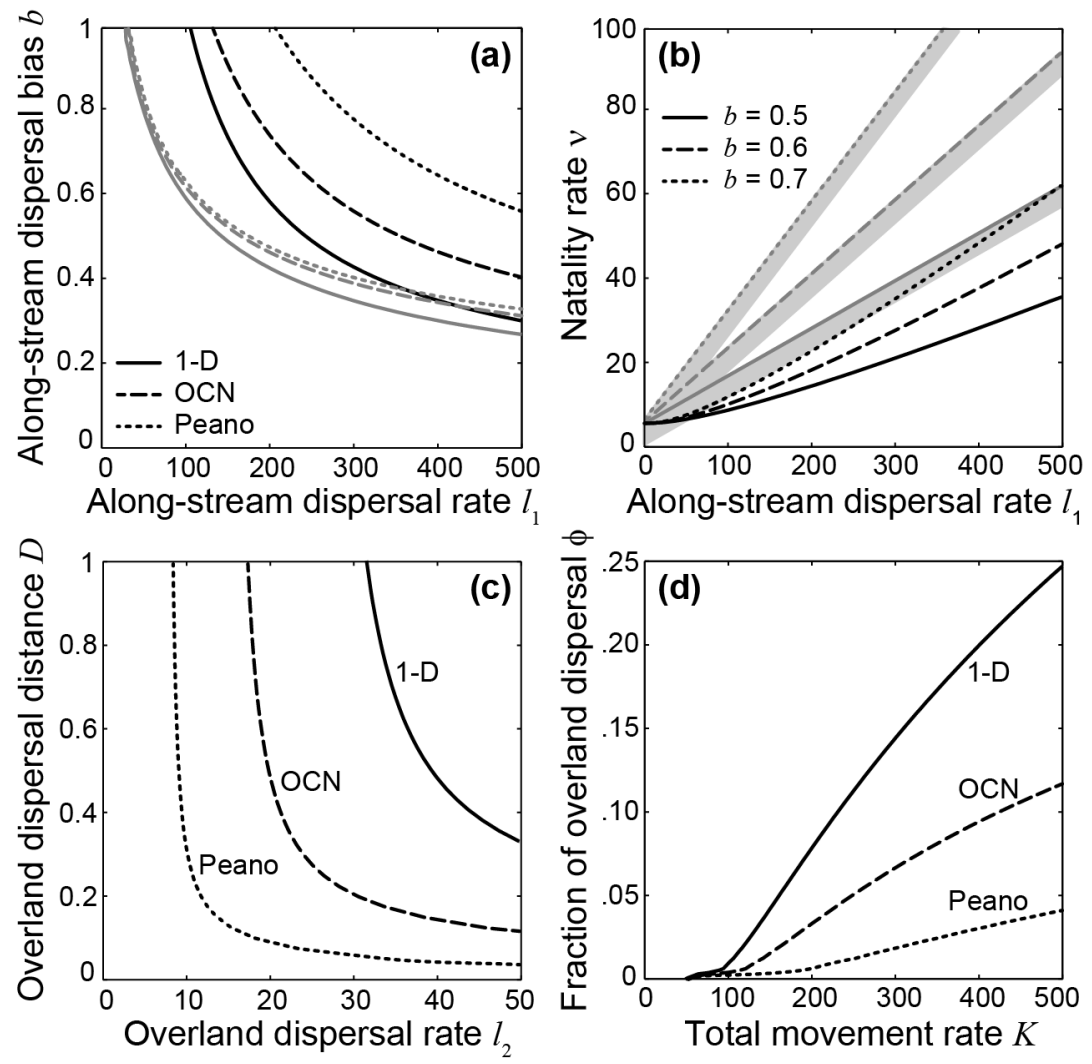

Figure 2: 

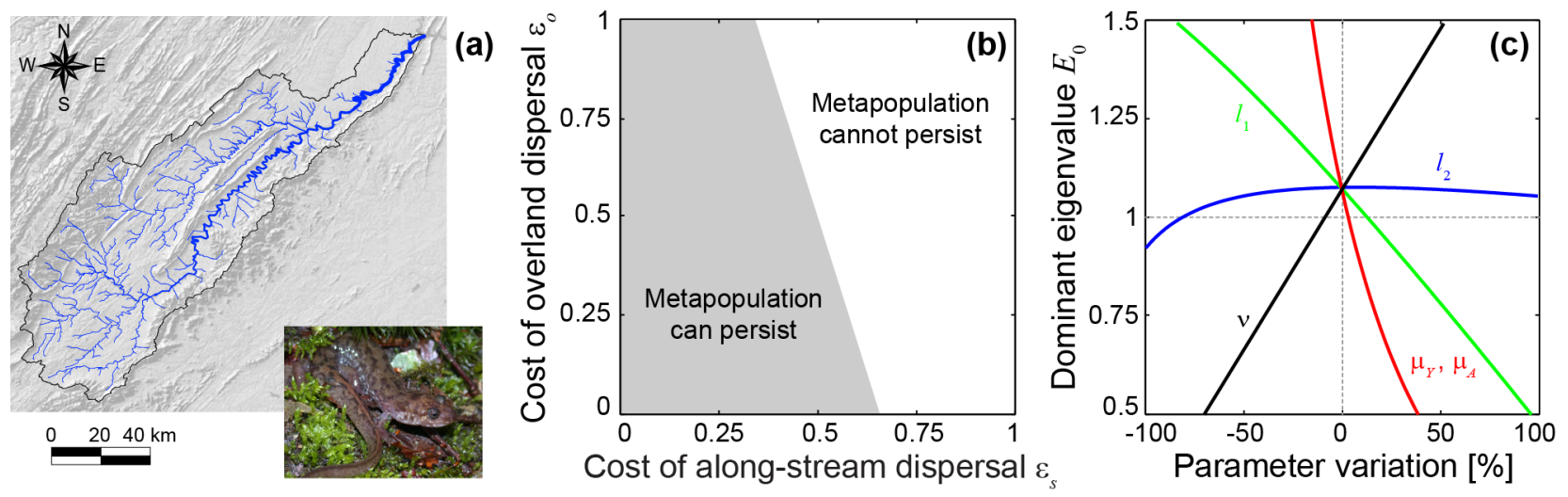

Figure 3:
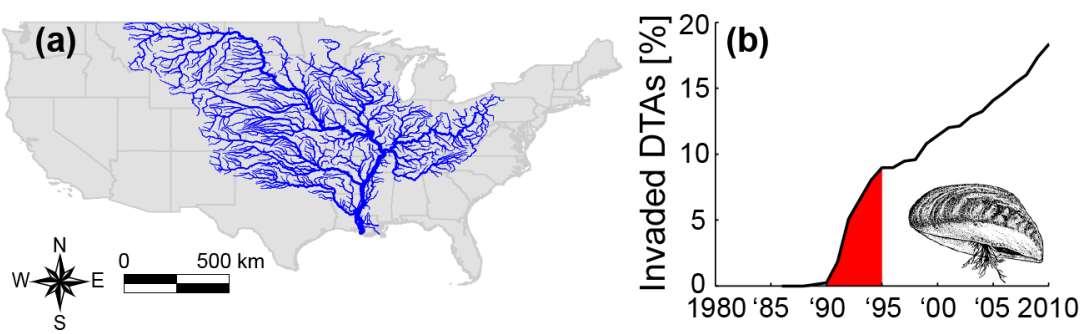

(c)
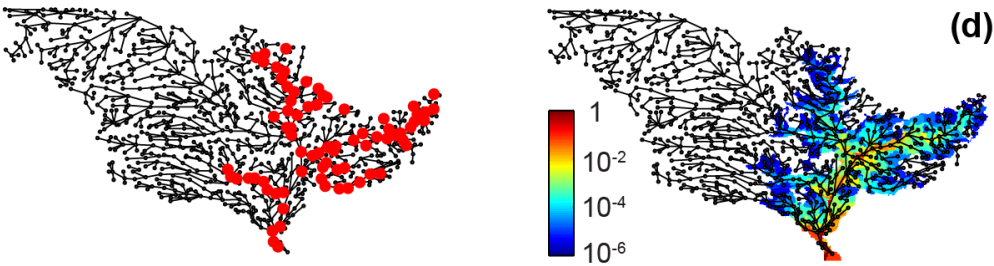

Figure 4: 